\title{
Cocaine induced midline destructive lesions
}

\author{
Edmiston $\mathbf{R}^{1 *}$, Chelliah $\mathbf{G}^{2}$ and Kumar $\mathrm{N}^{1}$ \\ ${ }^{1}$ Department of ENT, Wrightington Wigan and Leigh NHS foundation trust, UK \\ ${ }^{2}$ Department of Rheumatology, Wrightington Wigan and Leigh NHS foundation trust, UK
}

\section{Editorial}

Nasal crusting, nasal obstruction and epistaxis are common presentations to any ENT department and the importance of considering a systemic disease process is well practiced. Autoimmune, granulomatous, neoplastic, infective diseases and substance abuse can present in very similar ways with overlapping features necessitating a thorough diagnostic workup.

The effects of cocaine on the nasal mucosa are well documented with its vasoconstrictive properties resulting in tissue necrosis locally but it has also been found to have the potential to set off a systemic vasculitis. This process is thought to be as a result of the use of levamisole, a substance often cut into cocaine mixes which has immunomodulatory properties resulting in vasculitis and agranulocytosis [1].

A diagnostic dilemma arises when a patient with known substance abuse presents to clinic with significant nasal destruction and a clinician must be able to exclude a systemic vasculitis condition else a multisystem disease can go undiagnosed. Early diagnosis of such conditions is key as advanced disease limits the potential benefit of therapy [2].

In our case series we have developed a multidisciplinary pathway for patients presenting with nasal destructive changes and a prior history of cocaine use. We highlight the importance of first excluding a systemic vasculitis as well as treatment planning with input from rheumatology. We acknowledge the importance of assessing the disease extent with input from ENT colleagues who can also provide symptom control with an aim of restoring healthy mucosa. Finally, it is important to consider the disease implications such as the requirement for maxillofacial input for obturator fitting or reconstruction and audiologists to help provide symptom relief for hearing loss.

Throughout the diagnostic process there are clues that can guide clinicians as to the presence of systemic involvement. Simple tests of renal function and urinalysis can highlight obvious renal involvement, but the use of serological markers can sometimes complicate the picture.
Anti-neutrophil cytoplasmic antibodies (ANCA) offer a diagnostic aid when considering a diagnosis of granulomatous vasculitis conditions but as highlighted in our paper $56 \%$ of patients with CIMDL's are also c-ANCA positive with high levels of MPO antibodies. A negative result in this regard should also be reviewed with caution given the time for seroconversion with some patients with a true granulomatous vasculitis having negative results in early stages of the disease.

We have found that imaging enables an assessment of disease severity only and we suggest that there is no role for routine nasal biopsies in the absence of a suspicion of malignancy. Alternative biopsy sites such as renal in patients with abnormal renal function has been shown to have a much higher yield [3] and this may be considered in this cohort.

Our case series has identified that there is no single test that will confirm or dispute a diagnosis of systemic vasculitis and that the best approach to such patients is a multidisciplinary review to facilitate a prompt diagnosis and to avoid any treatment delays. We have highlighted the treatment options available for patients with CIMDL's including the use of immunomodulators, symptom relief techniques and reconstructive options.

In summary our case series reveals the pitfalls commonly encountered when trying to investigate such patients and we provide an investigative checklist to ensure all relevant specialties are involved to not only facilitate early treatment but also optimize symptom control.

\section{References}

1. Graf J, Lynch K, Yeh CL, Tarter L, Richman N, et al. (2011) Purpura, cutaneous necrosis and antineutrophil cytoplasmic antibodies associated with levamisole adulterated cocaine. Arthritis rheum 63: 3998-4001. [Crossref]

2. Lapraik C, Watts R, Bacon P, Carruters D, Chakrevarty K, et al. (2007) BSR and BHPR guidelines for the management of adults with ANCA associated vasculitis. Rheumatology (Oxford) 46: 1615-1616. [Crossref]

3. Devaney KO, Travis WD, Hoffman G, Leavitt R, Lebovics R, et al. (1990) Interpretation of head and neck biopsies in wegeners granulomatosis. A pathologic study of 126 biopsies in 70 patients. Am J Surj Pathol 14: 555-564. [Crossref]
Copyright: (C2018 Edmiston R. This is an open-access article distributed under the terms of the Creative Commons Attribution License, which permits unrestricted use, distribution, and reproduction in any medium, provided the original author and source are credited.
*Correspondence to: Edmiston R, Department of ENT, Wrightington Wigan and Leigh NHS foundation trust, UK, Tel: +86-20-81332566; E-mail: Rachel. edmiston@nhs.net

Received: September 07, 2018; Accepted: September 13, 2018; Published: September 17, 2018 